\title{
Uma Ferramenta para Análise da Comunicação Organizacional Através de Redes Sociais
}

\author{
Izabel Andion ${ }^{1}$, Manoel Mendonça ${ }^{2}$ \\ ${ }^{1}$ NUPERC - Núcleo de Pesquisa em Redes de Computadores - Universidade Salvador \\ (UNIFACS) \\ Caixa Postal - Salvador - BA - Brazil \\ ${ }^{2}$ NUPERC - UNIFACS - Salvador, BA - Brazil \\ icandiondlognet.com.br, mgmndunifacs.br
}

\begin{abstract}
This article describes a tool to analyze organizational communication networks. It models the network as a graph, implements Social Network Analysis (SNA) algorithms and allows the visual exploration of the graph. Compared to other SNA products, the developed tool incorporates semantic capability through the dynamic definition of attributes associated with the analyzed objects. The graph can be interactively explored and enables users to visually interpret relationships and roles over the modeled network. The tool was applied in a case study that identified communities of practice, communication patterns and key individuals over the modeled network.
\end{abstract}

Resumo. Este artigo descreve uma ferramenta para a análise de redes de comunicação organizacional. Ela modela a rede com um grafo, implementa algoritmos de Análise de Redes Sociais (SNA) e permite exploração visual do grafo. Comparada a outros produtos de SNA, a ferramenta desenvolvida incorpora mais semântica às análises através da definição dinâmica de atributos aos objetos analisados. Os grafos podem ser explorados interativamente permitindo aos usuários interpretarem visualmente relacionamentos e papeis na rede modelada. O ferramental foi aplicado em um estudo de caso que identificou comunidades de prática, padrões de comunicação e indivíduos responsáveis pela dinamização da rede analisada.

\section{Introdução}

Em meio à velocidade, variedade e complexidade crescente de informações e conhecimentos que se criam e se transformam na sociedade globalizada, as organizações e os indivíduos passam por uma intensa mudança na sua forma de aprendizado. Neste ambiente movido pela agilidade em adquirir novos conhecimentos, a rede de contatos que um indivíduo possui o auxilia na obtenção de alternativas e soluções para novos problemas. Uma teia de contatos bem estabelecida e bem dimensionada permite que o indivíduo tenha uma maior agilidade na resolução de problemas, na busca de conselhos e novas idéias. Para planejar e gerenciar de forma pró-ativa, obtendo uma maior sintonia e participação da cadeia de colaboração, a gerência necessita de conhecimento de como a organização funciona e se comunica. A compreensão do papel e da importância que 
cada colaborador desempenha em uma rede corporativa tanto formal quanto informal, permite uma visão eficiente do capital humano e social disponível em uma organização.

Análise de Redes Organizacionais se propõe a aplicar as técnicas da Análise de Redes Sociais (SNA) na organização. SNA tem como objetivo descobrir os padrões de interação entre as pessoas, visualizando seus movimentos e contatos.

O uso de imagens visuais onde a comunicação organizacional ou parte dela é apresentada como um grafo que se modifica em diversos cenários dependendo das dimensões e critérios analisados, possibilita uma visão crítica e auxilia na investigação e entendimento de comportamentos adotados pela comunidade. Métricas são aplicadas às interações entre as pessoas ou grupos, visando encontrar padrões de comportamento que auxiliam no entendimento dos interesses comuns e um melhor reconhecimento de suas práticas, revelando o trabalho real de uma organização.

Uma grande contribuição deste ferramental está em identificar pessoas que exercem influência na comunidade como líderes, formadores de opiniões e especialistas emergentes para que possam melhor compartilhar seus conhecimentos e competências, socializando suas experiências e aprendizados, adicionando capital social ao capital humano, acrescentando contatos ao conteúdo, ajudando numa efetiva identificação de padrões de colaboração e crescendo a rede de conhecimentos [Krebs 1998].

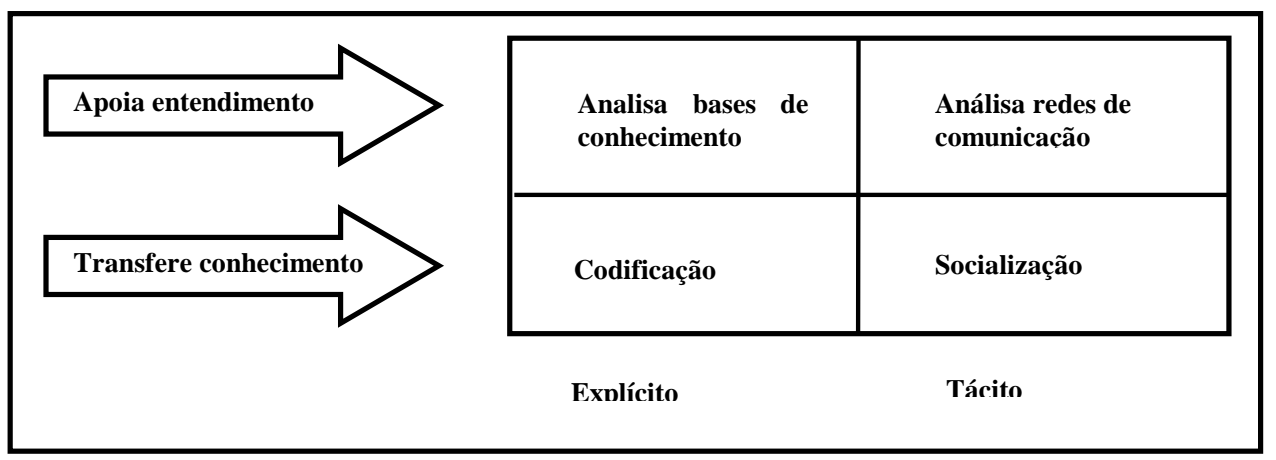

Figura 1. Categorização de ferramentas para Gestão do Conhecimento

A Figura 1 contextualiza o domínio do trabalho desenvolvido. Nela observam-se dois níveis de ferramentas: as que possibilitam a transferência de conhecimento e as que apóiam o entendimento e análise do uso do conhecimento.

As colunas indicam se o conhecimento transferido é explícito ou tácito [Nonaka 1997]. A transferência do conhecimento explícito se dá através das ferramentas de codificação que disponibilizam informações em bases de dados para acesso coletivo. Já o conhecimento tácito é trocado através de ferramentas de socialização que propiciam a comunicação e o compartilhamento de experiências com: correio eletrônico, chats, workgroup, workflows, call centers, helpdesk, entre outros [Hansen 1999].

O segundo nível diz respeito a ferramentas que analisam a utilização do primeiro nível. Na análise das ferramentas de codificação pode-se avaliar a freqüência de uso, necessidade de atualização, feedback de usuários, enfim, avaliar a utilização efetiva do conhecimento armazenado. $\mathrm{Na}$ análise das redes de comunicação avalia-se o comportamento de comunidades, a intensidade da comunicação e assuntos de interesse. 
A proposta deste artigo se enquadra na análise destas redes e está fortemente ancorada na teoria de Análise de Redes Sociais $(S N A)$ e em técnicas de exploração visual de dados. Estas ferramentas não enfatizam a codificação, classificação e armazenamento de conhecimento, mas sim o mapeamento e análise das interações sociais onde existem trocas de informações, conhecimentos, idéias e conselhos.

\section{Modelando a comunicação organizacional através de redes sociais}

A utilização de $S N A$ em organizações tem sido objeto de investigação, pois um valioso aspecto do conhecimento humano, o capital social, está inserido nas relações sociais. $\mathrm{O}$ conceito de capital social se baseia no valor das conexões entre os indivíduos de um grupo social para fins produtivos. É a riqueza que pode derivar de contatos, ligações e da capacidade de trabalhar bem com outras pessoas. Várias teorias e trabalhos sobre gerenciamento organizacional tentam explicar através do capital social como ligações pessoais ou posição na rede interferem no significado de poder, liderança, mobilidade, desempenho individual, criatividade e desempenho em grupo [Borgatti 2003].

O mapeamento da rede informal de comunicação consiste de mostrar como os fluxos de informações fluem na organização através de seus colaboradores, apontando elementos que influem neste processo de forma positiva ou negativa, criando assim oportunidades para corrigir e melhorar este ambiente a fim de propiciar uma maior troca e disseminação de conhecimentos entre os indivíduos.

\subsection{Analise de Redes de Sociais (SNA)}

O assunto SNA é interdisciplinar, ele engloba diversos campos do conhecimento acadêmico como: Sociologia, Psicologia Social, Antropologia, Estudos de Organização, Estatística, Matemática e Computação [Borgatti 2003]. SNA se baseia fortemente na teoria dos grafos. Voltada para organização, ela olha para o sistema de relacionamento humano como um sistema interconectado de nós e arestas, onde os nós são as pessoas ou grupos (atores) e as arestas (ligações) seus relacionamentos que acontecem através das trocas constantes de fluxos de informações e conhecimentos [Krebs 1998].

Existem três visões possíveis para a análise das características estruturais de uma rede social [Hanneman 2001], [Warserman 1999], [Scott 2000]. A primeira examina a rede como um todo, nesta análise, são avaliadas métricas que dizem respeito à composição da rede e ao grau de interação entre seus componentes. As principais métricas utilizadas nesta visão são:

- Tamanho da rede: o total de atores e ligações. Quanto maior for uma rede, mais difícil ficará conhecer os outros e trocar informações;

- Densidade da rede: a relação entre o total de ligações existentes e o total de ligações possíveis;

- Diâmetro da rede: a maior distância entre dois nós, indicando quantos atores no máximo deverão ser alcançados para se ligar os atores extremos da rede;

- Distância média: o valor médio referente a quantos atores no mínimo um ator tem que passar para chegar a todos os outros atores da rede. 
A segunda visão foca cada ator e seu papel na estrutura da rede através das métricas de centralidade. Estas métricas indicam a influência que o indivíduo exerce na manutenção e na expansão da rede. As principais medidas de centralidade são:

- Centralidade de grau mede a quantidade de ligações de um ator. Quanto mais ligações um ator possui, maior suas oportunidades de escolha e, conseqüentemente, sua autonomia em relação aos outros. Para redes que levam em consideração a direção das ligações, grau de entrada representa prestígio e popularidade, enquanto que grau de saída representa expansividade e influência.

- Centralidade de proximidade mede o número mínimo de passos que um ator deve empreender para entrar em contato com todos os outros atores. Quanto mais central for um ator, mais rapidamente ele entra em contato outros. Isto mede a independência do ator em relação ao controle exercido por outros.

- Centralidade de intermediação se baseia no controle exercido por um ator sobre as interações entre dois outros atores. Desde que dois atores não sejam vizinhos, eles dependem de outros atores do grupo para realizar suas trocas. A posição de intermediação determina influência de um ator. Atores que possuem uma alta intermediação têm uma boa visibilidade do que está acontecendo na rede.

A terceira visão é uma variante da primeira. Nela se analisa a rede de um ator específico, rede egocêntrica. São avaliados o ator em foco e as ligações com seus vizinhos e entre eles, buscando entender a influência do grupo sobre o indivíduo.

\subsection{Analise Visual de Grafos}

Visualização de dados permite que informações complexas sejam apresentadas através de metáforas visuais, melhorando a nossa capacidade de interpretação destes dados.

As imagens computacionais e as métricas baseadas em conceitos matemáticos são os dois fatores responsáveis pelo crescimento em pesquisas de redes sociais. A teoria dos grafos trouxe a construção de modelos estruturais mais genéricos e o aumento da disponibilidade dos computadores permitiu a produção de imagens automáticas. Atualmente as ferramentas usam cores, animação e permitem o usuário interagir com as imagens que recebem. Através da evolução destas ferramentas as imagens se tornaram um instrumento crítico e fundamental no auxílio das investigações, entendimento e comunicação dos pesquisadores em comportamentos das redes sociais [Freeman 2001].

Uma ferramenta para ser considerada de exploração visual de dados deve atender a requisitos básicos como: fazer uso de atributos visuais como forma, cor, tamanho para produzir cenas facilmente interpretáveis por seres humanos; possibilitar a navegação interativa na tela visual, permitindo zoom, re-posicionamento e varreduras sobre a área exibida; permitir o controle interativo dos formatos de apresentação e de atributos visuais exibidos; e permitir o detalhamento dos itens exibidos [Mendonça 2001].

Apesar de atualmente existirem várias ferramentas para $S N A$, elas não possuem uma adequada visualização interativa. Existe pouca semântica associada aos objetos representados. Os resultados das métricas são apresentados normalmente em estatísticas, sem refletir mudanças na cena visual. Na próxima seção será apresentada uma ferramenta que incorpora estas funcionalidades. 


\section{A Ferramenta FlowMiner}

A ferramenta FlowMiner objetiva trazer três contribuições importantes ao ferramental atualmente existente de análise de redes de comunicação organizacionais:

1. Ela aumenta o conteúdo semântico da rede estudada: a ferramenta adiciona o conceito de "conteúdo transacional" ao modelo de dados que normalmente se baseia somente em atores e ligações. Os conteúdos transacionais são os recursos materiais e não materiais trocados pelos atores através das ligações. Neste modelo de dados os objetos atores e fluxos passam a ser qualificados e categorizados por um número diverso e dinâmico de atributos. As ligações são tratadas como canais direcionados através dos quais trafegam os fluxos de comunicação. Para materializar esta abordagem, os dados coletados são armazenados em um modelo de dados relacional expansível. Atributos qualificadores dos objetos podem ser codificados conforme o tipo da organização estudada.

2. Ela utiliza de técnicas de visualização para representar os resultados encontrados. $O$ uso de uma metáfora visual consistente permite que os resultados sejam apresentados de forma simples e de fácil compreensão. A ferramenta permite que objetos e seus atributos sejam mostrados, retirados ou centralizados na cena visual com um simples clique do mouse. Os cenários podem ser dinamicamente montados e apresentados em grafos, e estes grafos podem sofrer aproximações, rotações e alteração de seu raio de exibição. A apresentação das arestas em diversos tons de cores mostra a qualidade das ligações entre os atores, enfatizando ligações com maior intensidade de fluxos. Cores também são utilizadas para apresentar os resultados das métricas sobre a cena visual, realçando com tons diferenciados os atores mais bem pontuados para uma determinada consulta.

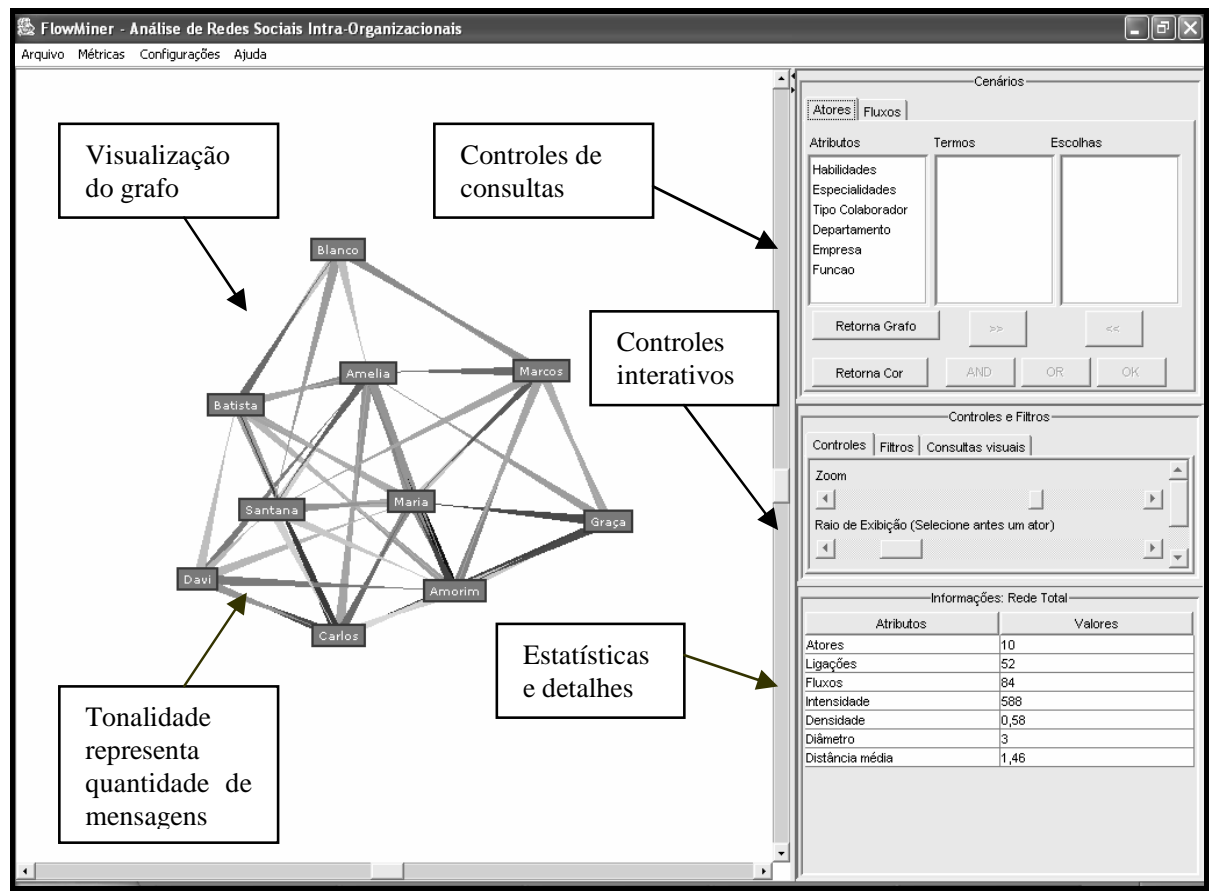

Figura 2. Tela principal da FlowMiner.

3. Ela utiliza interatividade através de consultas dinâmicas aos vários atributos que qualificam os objetos. Atores e fluxos podem ser selecionados na cena visual através da combinação de valores dos seus atributos. Este processo permite a montagem interativa 
e dinâmica de grafos que representam comunidades de prática dentro da organização. Sobre as comunidades que formam uma determinada cena visual, podem ser aplicadas as métricas codificadas para analisar a rede social.

A ferramenta foi desenvolvida em Java. Para exploração visual de dados foram construídos diversos controles a partir do TouchGraph, um framework para visualização de grafos [TouchGraph 2004]. Sua tela principal está na Figura 2. Ela contém quatro painéis distintos. $\mathrm{O}$ primeiro apresenta o grafo representando o mapa de comunicação estudado. $\mathrm{O}$ segundo representa as opções de controle de consultas, onde o usuário através da seleção de valores de atributos de atores ou fluxos pode modificar o cenário apresentado. A terceira área mostra os controles de interações que são compostos de controles de aproximação, raio exibição e rotação; filtros por atributos quantitativos e consultas visuais para mostrar visualmente distribuição de valores por atributo. O quarto painel mostra as tabelas de detalhes sob demanda que são preenchidas com os resultados das consultas feitas. A subseção a seguir mostra as abordagens de uso da ferramenta.

\subsection{Análise da Rede Total}

A análise da rede total fornece uma visão global da rede estudada, mostrando a estrutura de comunicação entre seus atores. Sempre que um grafo é apresentado no primeiro painel, no quarto painel mostrará as métricas relativas à estrutura geral do grafo. No mapa apresentado na Figura 2 podemos observar que a rede possui 10 atores com 52 ligações por onde trafegam 84 fluxos de intensidade 588 (total de mensagens). O fluxo é a consolidação de mensagens por ligação, atributo e valor. A densidade deste mapa é alta, mostrando que $58 \%$ das conexões possíveis foram realizadas. Esta é uma rede densa e pouco hierarquizada. A distância média apresentada $(1,46)$ confirma a coesão da rede, pois cada ator alcança qualquer outro em média com uma ligação e meia.

Podemos identificar visualmente também as ligações que possuem maior intensidade de fluxo através sua coloração mais forte. Efetuando um clique do mouse em qualquer objeto do grafo serão apresentadas na quarta área da tela as informações detalhadas do objeto em questão.
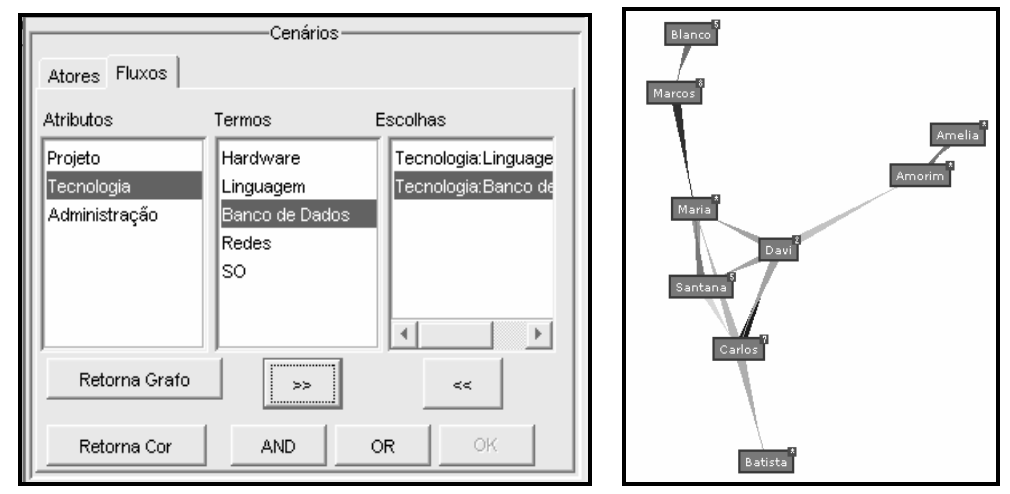

Figura 3. Seleção de atributos de fluxo e o grafo gerado.

\subsection{Análise da Rede após Consulta}

No segundo painel, responsável pelos controles de consultas, existe duas abas: Atores e Fluxos. Escolhendo uma, a lista Atributos será preenchida com os atributos encontrados na base de dados para o elemento escolhido. Escolhendo um dos valores, a lista de 
Termos será preenchida com o domínio do atributo. O usuário poderá fazer consultas escolhendo termos do domínio e utilizando operadores lógicos para executá-la.

Na Figura 3 o cenário escolhido foi por Fluxos sobre o assunto Tecnologia e os termos: Banco de Dados ou Linguagem. Após a execução da seleção, a consulta é propagada para cena visual deixando no grafo apenas os elementos que satisfazem os fluxos especificados. Agindo desta forma, múltiplos cenários podem ser montados com poucos toques no mouse.

\subsection{Análise da Rede por Ator}

Várias medidas podem ser usadas para se analisar o papel que cada ator desempenha em uma determinada rede. As principais medidas usadas na ferramenta são discutidas na seção 2.1.

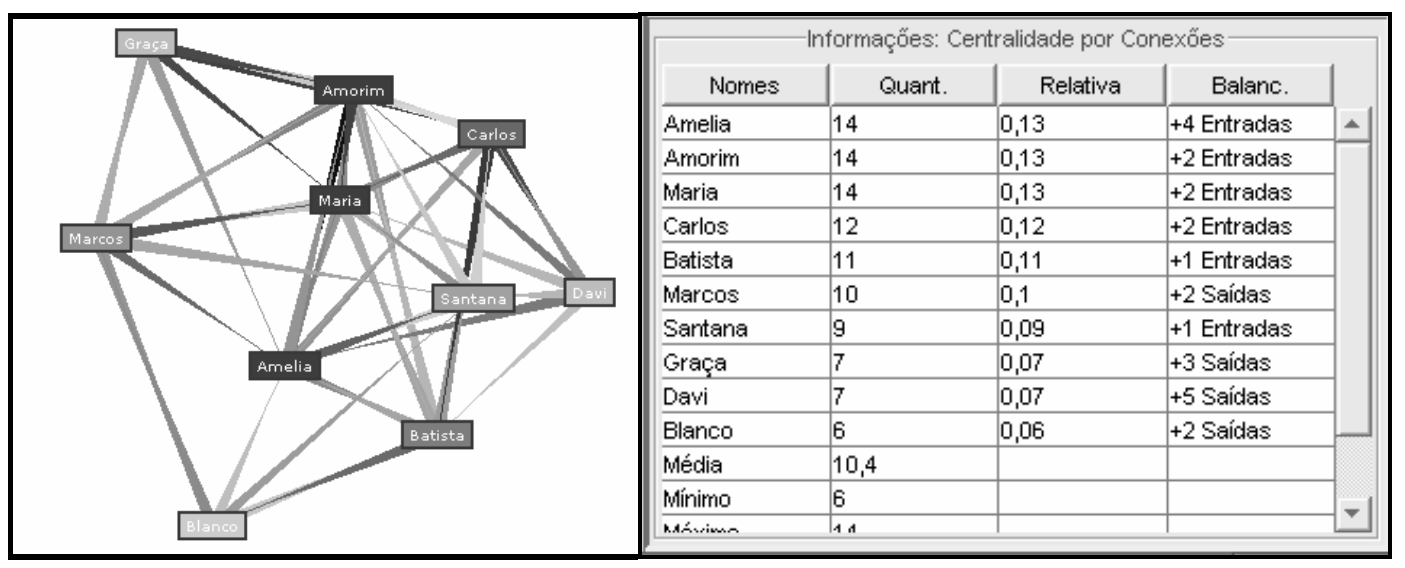

Figura 4. Grafo e detalhes gerados para a métrica Centralidade por Conexões.

A Figura 4 ilustra o que acontece na tela visual quando uma destas métricas é selecionada. A tonalidade de cada nó varia com o valor da medida calculada. Cores mais fortes representam valores maiores. $\mathrm{O}$ quarto painel mostra os valores encontrados de cada ator para a medida selecionada, trazendo também um sumário de seu valor mínimo, máximo, média, variância e desvio padrão da métrica.

\subsection{Estudo de Caso}

Foi desenvolvido um estudo de caso, onde a ferramenta analisou a comunicação entre colaboradores de uma empresa de consultoria de informática para resolver problemas e esclarecer dúvidas sobre manutenção de sistemas (HelpDesk). Os dados coletados corresponderam a quatro meses de comunicação entre uma população de 67 indivíduos envolvidos em processo de manutenção de sistemas. Durante este período trafegaram na rede 1734 mensagens de assuntos variados.

Após a coleta, os dados passaram por processos de transformações até se tornarem aptos a inclusão na base de dados da ferramenta. Uma vez inserido os dados, foi executada a análise da rede social apresentada utilizando a ferramenta FlowMiner. A análise contou de quatro passos: análise da rede como um todo, análise por ator, análise dos cenários de comunidades e análise das redes egocêntricas.

Ao final da análise dos resultados apresentados, a ferramenta possibilitou determinar: a hierarquização da rede; necessidade de treinamento; padrões de 
comportamentos; identificação de indivíduos mais atuantes e maiores colaboradores por assunto.

\section{Considerações Finais}

FlowMiner é uma ferramenta voltada a visualização de redes sociais utilizando grafos como metáfora visual (MV). Esta MV é construída de forma navegacional utilizando o arcabouço TouchGraph e acessando uma base de dados específica. As principais contribuições da FlowMiner estão na possibilidade de atribuir semântica nos objetos representados e a utilização de vários controles de visualização e interação que facilitam o entendimento e a capacidade de inspeção dos dados. Através desses controles visuais é possível: ocultar ou exibir objetos, construindo cenas variadas através das consultas por atributos; aplicar métricas de $S N A$ sobre as cenas geradas; filtrar através de mudança de cores atributos quantitativos dos objetos; exibir sob demanda a distribuição dos valores dos atributos dos objetos da cena visual e manipular o grafo, mudando o posicionamento de seus elementos para melhorar a visualização.

O uso de SNA juntamente com as características de exploração visual e interatividade da ferramenta permitem e facilitam a combinação de elementos para o entendimento e a avaliação do modo de comunicação da organização estudada. Os padrões de comunicação dos atores que participam da rede são facilmente avaliados além de identificar indivíduos líderes ou que atuam como mediadores ou facilitadores do fluxo de informações de uma organização.

\section{Referências}

Borgatii, S and Foster P. (2003) "The Network Paradigm in Organizational Research : A Review and Topology.”, Journal of Management Vol. 29(6), p 991-1013.

Freeman, L. (2000) "Visualizing Social Networks", Journal of Social Structure (1)1.

Hanneman, R. (2001) "Introduction to Social Methods". Department of Sociology. Technical Report University of California, Riverside.

Hansen, M. and Nohia, N.; Tierney, T. (1999) "What's your strategy for managing knowledge?" In: Harvard Business Review on Organization Learning, Harvard Business Scholl Press, p.61-86

Krebs, V. (1998) "Mapping and Measuring Knowledge Creation, Re-use and Flow". http://www.orgnet.com/ fevereiro 2004

Mendonça, M. and Almeida, M. (2001) "Uso de Interfaces Abundantes em Informações para Mineração Visual de Dados”. IHC’2001.

Nonaka, I. and Takeuchi, H. (1997) "Criação de Conhecimento na Empresa: Como as empresas japonesas geram a dinâmica da inovação". Editora Campus, 358 p.

Scott, J. (2000) "Social network analysis: a handbook". Sage Publications. 208p.

TouchGraph (2004) TouchGraph. http://www.touchgraph.com/ fevereiro 2004.

Wasserman, S. and Faust, K. (1999) "Social Network Analysis: Methods and Applications". Cambridge University Press, 825p. 